\title{
Charge-Transfer Photodissociation of Adsorbed Molecules via Electron Image States
}

\author{
E.T. Jensen* \\ Physics Department, University of Northern British Columbia \\ 3333 University Way, Prince George B.C. V2N 4Z9, Canada
}

(Dated: September 3, 2021)

\begin{abstract}
The $248 \mathrm{~nm}$ and $193 \mathrm{~nm}$ photodissociation of submonolayer quantities of $\mathrm{CH}_{3} \mathrm{Br}$ and $\mathrm{CH}_{3} \mathrm{I}$ adsorbed on thin layers of $n$-hexane indicate that the dissociation is caused by dissociative electron attachment from sub-vacuum level photoelectrons created in the copper substrate. The characteristics of this photodissociation- translation energy distributions and coverage dependences show that the dissociation is mediated by an image potential state which temporarily traps the photoelectrons near the $n$-hexane-vacuum interface, and then the charge transfers from this image state to the affinity level of a co-adsorbed halomethane which then dissociates.
\end{abstract}

\section{INTRODUCTION}

One of the recurring themes in surface photochemistry on metal surfaces is the almost ubiquitous contribution that photoelectrons make to the observed photochemical processes. In particular, many phenomena have been interpreted as being the consequence of hot photoelectrons- those photoelectrons having energies between the Fermi and the vacuum levels of the system. In nanosecond timescale photochemistry on noble metal substrates, the system has sufficient time for many cycles of electron excitation, scattering and decay, so that the resultant electron energy distribution that results has a form $n(E) \propto\left(E-E_{F e r m i}\right)^{-2.5}$ at energies well above $E_{\text {Fermi }}[1$. This rather smooth energy distribution can interact with the relevant electron affinity levels of the adsorbate system. If one is particularly interested in the dissociation of molecules via dissociative electron attachment (DEA), then one would expect that the convolution of the $n(E)$ with the $\sigma_{D E A}(E)$ will yield a flux of desorbing species that arises from a relatively wide range of photoelectron energies (roughly the width of $\sigma_{D E A}(E)$ for energies a few eV above $E_{\text {Fermi }}$ ).

One can inquire if there are mechanisms that will modify this nascent energy distribution- in particular, can the photoelectron energy distributions be narrowed to be more selective in the dissociation of adsorbed species. A related question has to do with electron transport in thin molecular films and how the electrons in these films can interact with other species- again, are there physical systems that will be highly selective in the interactions between co-adsorbed molecules? One approach was first detailed on theoretical grounds by Rous [2] who considered how an incoming electron might couple with the electron affinity levels of a $N_{2}$ molecule near a metal surface to vibrationally excite the molecule. It was shown by application of a KKR calculation that the electron affinity level $\sigma_{v i b}(E)$ would display a strong enhancement by resonance with electron image states of the surface system.

*Electronic address: ejensen@unbc.ca
In order to form such an image state and have an image state lifetime sufficient to yield a significant enhancement, one requirement is that the surface have a bandgap at the relevant electron energy (typically E $0.5 \mathrm{eV}$ relative to $E_{\text {vacuum }}$ for the $n=1$ state), which does indeed occur for a number of low index metal surfaces. A practical complication is that these image states on clean metal surfaces have been found to be very sensitive to contamination effects- rather small concentrations of some adsorbates on metal surfaces have been found to open decay channels that suppress the image state 3. A different approach is to take advantage of image states that can be formed at dielectric surfacesthese were first described in detail by Cole and Cohen 4 and were subsequently observed using microwave spectroscopy on $l$-He surfaces [5]. More recent work on image states at dielectric thin films has been in the context of characterizing the energies and lifetimes of these states using femtosecond two-photon photoemission (fs-2PPE) spectroscopy. In general terms, molecules having negative electron affinities (i.e. repulsive for low energy electrons) in thin layers form a bandgap that encompasses the image state energies (i.e. the energy region just below $\left.E_{\text {vacuum }}\right)$. In qualitative terms, the decay of these electron image states is dominated by the transmission probability through the barrier, a barrier that increases the lifetimes roughly exponentially with film thickness. There is now a substantial body of work on the effect of thin films interacting with intrinsic metal image states for both the case of available conduction band states at the image state energies (image state resonance, e.g. Kr and $\mathrm{Xe}$ on $\mathrm{Cu}(100)[6]$ ) and pure image electron barrier layers (e.g. thin alkane layers [7]) on various metal surfaces. There has also been work on coupling of a negative ion resonance for $\mathrm{O}_{2}$ with an image state resonance formed for $\mathrm{Xe} / \mathrm{Cu}(111)$, in which the $\mathrm{O}_{2}$ anion state couples to the Xe conduction band, with a strong layer dependence for the anion state lifetime 8 .

For the present case, a schematic image potential at a 4ML $n$-hexane on $\mathrm{Cu}(110)$ is shown in Fig. 1. We have used the modified dielectric continuum model of Hotzel et al $[9$ to describe the image potential at the $n$-hexanevacuum interface, and physical values similar to those chosen in recent fs-2PPE studies of this system (for $n$ - 


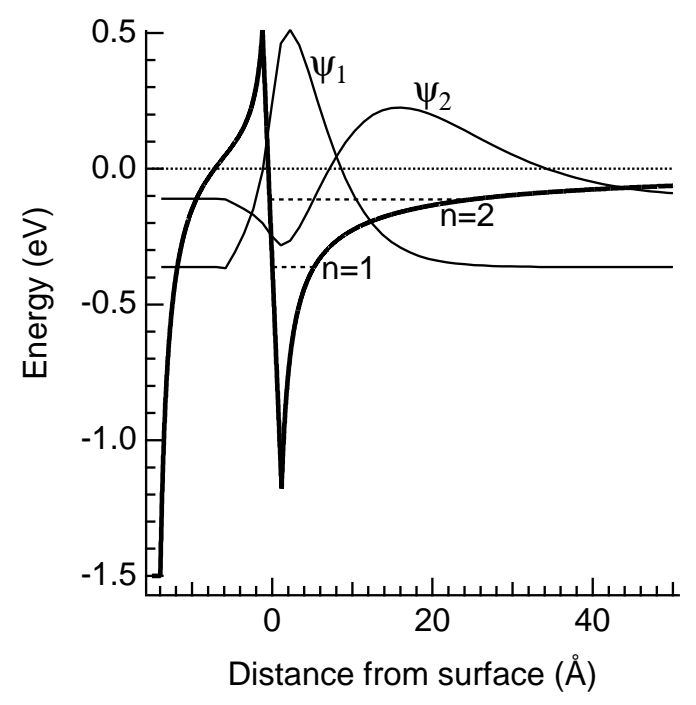

FIG. 1: Schematic image potential and 2 lowest bound states for approximately $4 \mathrm{ML}(15 \AA)$ of $n$-hexane on $\mathrm{Cu}(110)$ obtained using a dielectric continuum model. The $n$-hexanevacuum interface is at $0 \AA$ and the $\mathrm{Cu}(110)$ surface is located at $-15 \AA$. The negative electron affinity of $n$-hexane $(\mathrm{EA} \approx-$ $0.2 \mathrm{eV}$ ) creates a barrier at the surface which can trap electrons in the image states. The $\mathrm{Cu}(110)$ substrate has no bandgap at the image state energies, so electrons that tunnel through the $n$-hexane barrier can enter unoccupied metal states directly.

hexane, EA=-0.20eV, $\left.\epsilon_{r}=2.0\right)[7]$. The lowest lying image states have binding energies of $0.36 \mathrm{eV}(n=1)$ and $0.11 \mathrm{eV}(n=2)$. In the case of the $\mathrm{Cu}(110)$ substrate used in this work there is no intrinsic surface bandgap near $\bar{\Gamma}$ at these energies, so that image state electrons that tunnel through the $n$-hexane barrier layer will simply enter $\mathrm{Cu}$ conduction band states. In the experiments described below, a submonolayer of 'detector' molecules $\left(\mathrm{CH}_{3} \mathrm{Br}\right.$ or $\mathrm{CH}_{3} \mathrm{I}$ ) are adsorbed on top of the $n$-hexane layer. If the dissociative attachment cross section for these adsorbed molecules has a substantial overlap with the image state energy and the image state has sufficient lifetime, we expect that there would be image state mediated DEA observed. Fig. 1 also shows that the largest spatial overlap between the image states and an adsorbed molecule will occur for the $n=1$ image state- for higher $n$ image states, the wavefunction becomes increasingly weighted away from the surface. From fs- $2 \mathrm{PPE}$ experiments, it is also found that the $n=1$ image state has a substantially higher population than the $n=2$ and higher states [10, so one would expect dissociation to occur primarily from the $n=1$ state.

For $\mathrm{CH}_{3} \mathrm{Br}$ and $\mathrm{CH}_{3} \mathrm{I}$ in the gas phase, DEA occurs for low energy electrons with a threshold electron energy related to the curve crossing of the neutral ground state potential with the anion state 11, 12. The photodissociation of these halomethanes at metal surfaces and the role of photoelectron mediated DEA has been studied by a variety of workers $13,14,15$. When these molecules are adsorbed at a metal or dielectric surface, the anion state is shifted downward in energy and the threshold electron energy for DEA decreases and the survival probability for the anion increases, so that in general the peak DEA cross section is at lower electron energy and has a substantially larger magnitude. These effects are well known and have been previously reported in the literature (e.g. see Ref. [16]). In the case of $\mathrm{CH}_{3} \mathrm{Br}$ and $\mathrm{CH}_{3} \mathrm{I}$, the lowering of the anion potential by $\sim 1 \mathrm{eV}$ will result in the threshold electron energy for DEA at or close to $0 \mathrm{eV}$ and the peak DEA cross section at very low electron energy. At the surface, these correspond to electron energies below the vacuum level [17, 18], and can be accessed by the hot photoelectrons discussed above and, as will be discussed in the present work, will also have energetic overlap with electron image states at the surface.

\section{EXPERIMENTAL METHODS}

Experiments were performed using an ultra-high vacuum surface science and photochemistry apparatus that has been described previously [19. We have utilized both $\mathrm{Cu}(100)$ and $\mathrm{Cu}(110)$ single crystal substrates in this work. The samples are heated to $915 \mathrm{~K}$ by electron bombardment for cleaning and cooled to $\sim 92 \mathrm{~K}$ using liquid nitrogen, the temperature at which the experiments were performed. Sample cleanliness and order is monitored by Auger electron spectroscopy and low energy electron diffraction measurements. Neutral products from surface photodissociation travel $185 \mathrm{~mm}$ to pass through a $4 \mathrm{~mm}$ diameter aperture to a differentially pumped Extrel quadrupole mass spectrometer with an axial electron bombardment ionizer. The sample to ionizer distance is $203 \mathrm{~mm}$. Ions created in the ionizer then travel to the quadrupoles and are mass selected, in the present experiments using $\mathrm{m} / \mathrm{q}=15 \mathrm{amu}\left(\mathrm{CH}_{3}^{+}\right)$. Selected ions are detected by a conversion dynode and electron multiplier in pulse counting mode and the pulses are then passed through a fast preamplifier. The particle arrival pulses are time recorded using a multichannel scaler (MCS) that begins counting prior to the initiating laser pulse, with a typical time-of-flight (TOF) spectrum using 1000 $1.0 \mu$ s time bins, and summing the counts from 1000 laser pulses.

The laser pulses ( $~ 5 \mathrm{~ns}$ duration) are produced by a small excimer laser (MPB PSX-100) operating at 20Hz. Both unpolarized and linearly polarized light has been used in this work. To create polarized light, the beam passes through a birefringent $\mathrm{MgF}_{2}$ crystal to separate pand s-polarized light, which can then be directed at the sample. Unless otherwise stated, p-polarized laser pulses were used in the present study. Various laser wavelengths are produced by selection of different laser gases: 193nm (ArF), 248nm (KrF) and 308nm (XeCl). Pulse energies varied for the different laser wavelengths, but the fluence on the sample were less than $1 \mathrm{~mJ} / \mathrm{cm}^{2}$. The laser pulses were collimated using a $4 \mathrm{~mm}$ diameter aperture and were 
unfocused on the sample. The laser light is incident upon the sample at an angle of $45^{\circ}$ from the mass spectrometer axis- when the $\mathrm{Cu}$ sample is aligned to collect mass fragments along the surface normal, the light is incident at $45^{\circ}$. Angular distributions of the photofragments were measured by rotating the sample with respect to the mass spectrometer axis, which also changes the incident angle of the laser light. In the present experiments the effect of changing the incident light angle is small and its consequence on photoelectron generation is well understood.

The liquids used for dosing in the present work: $n$ hexane (Aldrich $\geqslant 99.5 \%$ ) and $\mathrm{CH}_{3} \mathrm{I}$ (Aldrich 99.5\%)were transferred to a glass and teflon gas-handling system and degassed by freeze-pump-thaw cycles. The $\mathrm{CH}_{3} \mathrm{Br}$ (Aldrich 99.5\%) was used as delivered from a lecture bottle. Room-temperature vapor pressures were used for gas dosing through a precision leak valve that backfilled the chamber. Gas doses are described using uncorrected ion gauge readings. Gas doses that correspond to monolayer coverages were calibrated using temperature programmed desorption measurements or from prior experience with the selected molecule in other experiments.

\section{RESULTS AND DISCUSSION}

A set of TOF spectra using $248 \mathrm{~nm}(h \nu=5.0 \mathrm{eV})$ laser pulses for $\mathrm{CH}_{3} \mathrm{Br}$ adsorbed on $\mathrm{Cu}(110)$ are shown in Fig. 2. In the gas-phase, $\mathrm{CH}_{3} \mathrm{Br}$ has a low cross section for direct photodissociation at this wavelength, so it is expected that DEA by photoelectrons will be the dominant dissociation mechanism. This expectation is supported by the observed TOF spectra. In Fig. 2(a) the TOF spectrum for a submonolayer of $\mathrm{CH}_{3} \mathrm{Br}$ adsorbed on a $4 \mathrm{ML} n$-hexane layer on $\mathrm{Cu}(110)$ shows the single narrow feature that is ascribed to the CT-DEA via an image state intermediate. That this feature is caused by photoelectrons is supported by the spectrum of Fig. 2 (b), in which the substrate photoelectron yield is reduced due to the addition of atomic iodine to the $\mathrm{Cu}$ surface, which forms a $\mathrm{c}(2 \times 2) \mathrm{Cu}(110)-\mathrm{I}$ surface 20, 21 . This iodization largely suppresses the $\mathrm{CH}_{3} \mathrm{Br}$ dissociation signal, though a small peak at the same flight time is just visible. That this small feature is observed at the same flight time (i.e. same $\mathrm{CH}_{3}$ translational energy) supports the notion of an image state intermediate at the $n$-hexane surface, since one would expect that the photoelectron energy distribution would be altered by the iodization. In general, changes in the photoelectron energy distribution will be reflected in the TOF spectrum if the DEA occurs via a resonance at an energy where the photoelectron distribution is altered. In Figs. 2 (c) and (d) the $\mathrm{CH}_{3} \mathrm{Br}$ image state mediated dissociation to differs from that of $1 \mathrm{ML} \mathrm{CH}_{3} \mathrm{Br}$ on clean $\mathrm{Cu}(110)$ (Fig. 2(c)) and $2 \mathrm{ML} \mathrm{CH}_{3} \mathrm{Br}$ on $\mathrm{Cu}(110)-\mathrm{I}$ (Fig. 2(d)). In these latter spectra, the peak of the TOF spectrum is shifted and the TOF peaks are broader than that of Fig. 2(a). The absolute width in energy (FWHM) for the peak in Fig. 2(a) is $0.18 \mathrm{eV}$, while for Figs 2 (c) and (d) the widths are 0.28 and $0.24 \mathrm{eV}$ respectively. The relative peak widths $\left(\Delta E / E_{\text {peak }}\right)$ are $0.32,0.62$ and 0.64 respectively, again highlighting the distinctly narrower translational energy distribution for dissociation via the image state electron mechanism. In particular, comparison of the spectra of Figs 2(a) and (d) for $\mathrm{CH}_{3} \mathrm{Br}$ on $\mathrm{Cu}(110)-\mathrm{I}$ is telling, since this $\mathrm{CH}_{3} \mathrm{Br}$ layer is known to be quite well orientationally ordered and can display very narrow TOF features 22. At $248 \mathrm{~nm}$, it appears that the width of the TOF feature for $\mathrm{CH}_{3} \mathrm{Br} / \mathrm{Cu}(110)-\mathrm{I}$ in Fig. 2(d) is dominated by the range of photoelectron energies that cause DEA, resulting in $\mathrm{CH}_{3}$ fragments of more widely varying kinetic energy. The narrow TOF spectrum of Fig. 2(a) for $\mathrm{CH}_{3} \mathrm{Br} /$ hexane $/ \mathrm{Cu}(110)$ is the first piece of evidence that the DEA is dominated by a very narrow range of incident electron energies, due to coupling with an image state that has captured a photoelectron which is then coupled to the $\mathrm{CH}_{3} \mathrm{Br}$ affinity level for DEA.

Time-of-flight spectra for $\mathrm{CH}_{3} \mathrm{Br}$ using $193 \mathrm{~nm}$ $(h \nu=6.40 \mathrm{eV})$ are shown in Fig. 3. In contrast to the $248 \mathrm{~nm}$ case, at $193 \mathrm{~nm} \mathrm{CH} \mathrm{CH}_{3} \mathrm{Br}$ is known to have a large cross section for direct photodissociation in the gasphase 23. and at surfaces 22. It is therefore somewhat surprising to find that for $0.1 \mathrm{ML}$ of $\mathrm{CH}_{3} \mathrm{Br}$ on top of $4 \mathrm{ML}$ of $n$-hexane on $\mathrm{Cu}(110)$ that the same TOF distribution is seen using $193 \mathrm{~nm}$ light as for $248 \mathrm{~nm}$ (Fig. 3(a)). The peak in this TOF spectrum is at exactly the same flight time as for the $248 \mathrm{~nm}$ case, showing the same energy release in $\mathrm{CH}_{3}$ translation in spite of the $1.40 \mathrm{eV}$ higher photon energy. That the position and width of this TOF feature is unchanged shows that the electrons responsible for this dissociation have the same energy distribution and is further support for the image state mediated mechanism of photodissociation. At $193 \mathrm{~nm}$, as the $\mathrm{CH}_{3} \mathrm{Br}$ coverage in increased, the total $\mathrm{CH}_{3}$ yield increases but a new feature at $44 \mu \mathrm{s}$ flight time emerges (Fig. 3 (b)) and at higher $\mathrm{CH}_{3} \mathrm{Br}$ coverage, dominates the spectrum. This feature is associated with the $193 \mathrm{~nm}$ direct photolysis of $\mathrm{CH}_{3} \mathrm{Br}$, as it peaks at the same flight time as that seen in a previous study [22]- a representative TOF spectrum is shown in Fig. 3(c) for comparison. Thus even at a wavelength at which $\mathrm{CH}_{3} \mathrm{Br}$ has a large direct photodissociation cross-section, the image state mediated dissociation dominates at low coverage. This is due to the large effective cross section for this process as the photoelectrons that are promoted to the image state are free-electron like parallel to the $n$-hexane surface, and can cause DEA in adsorbed species that are relatively distant from the location of the initial excitation. For example, for a trilayer of $n$-pentane on $\operatorname{Ag}(111)$, the measured $n=1$ image state lifetime is $17.6 \mathrm{ps}$ (and increasing by roughly $10 \times$ per added monolayer) [7, so that such an $m^{*}=1$ image state electron with $\sim 0.2 \mathrm{eV}$ of kinetic energy would have a mean free path length of roughly $4.6 \mu \mathrm{m}$. At higher $\mathrm{CH}_{3} \mathrm{Br}$ coverages (approaching 1 monolayer and above), the image state mediated process persists but the direct photodissociation mechanism becomes more competitive. 


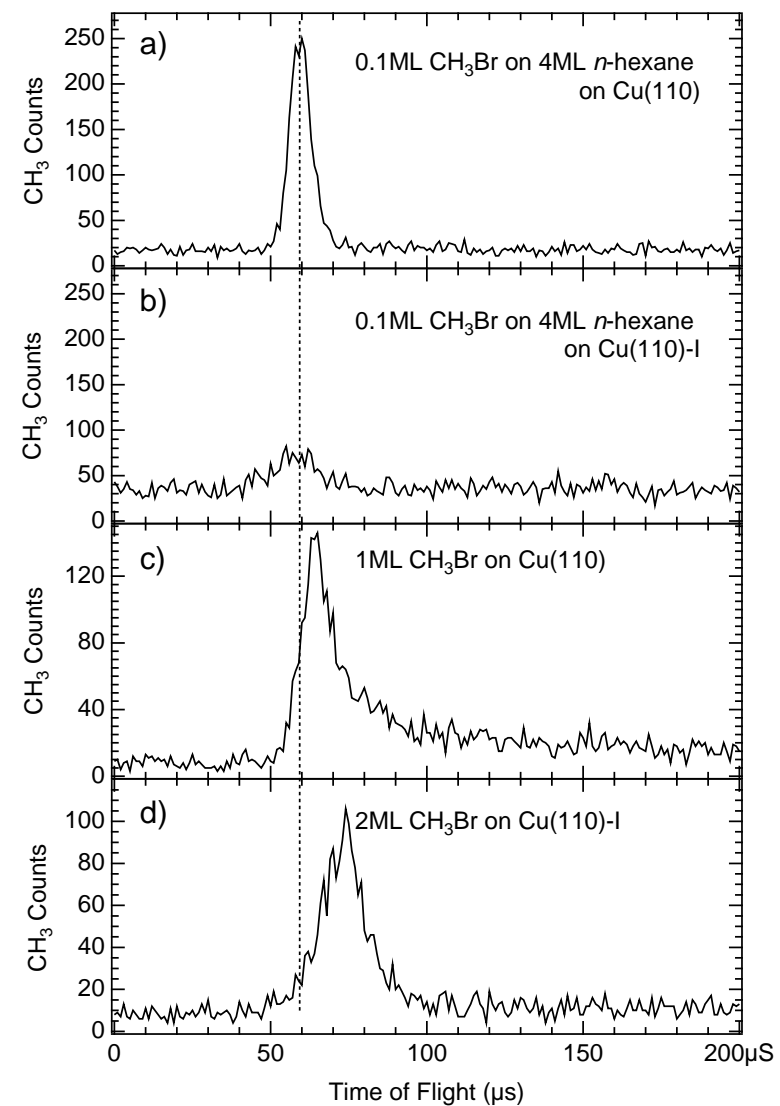

FIG. 2: Time-of-flight spectra for $\mathrm{CH}_{3} \mathrm{Br}$ obtained using 1000 pulses of $248 \mathrm{~nm}$ light. In (a) the signal is from a submonolayer (0.1ML) of $\mathrm{CH}_{3} \mathrm{Br}$ on top of $4 \mathrm{ML} n$-hexane on the $\mathrm{Cu}(110)$ substrate. Spectrum (b) is obtained in a similar situation to (a), except that the substrate is $\mathrm{c}(2 \times 2) \mathrm{Cu}(110)-\mathrm{I}$. The spectrum (c) is obtained from $1 \mathrm{ML}$ of $\mathrm{CH}_{3} \mathrm{Br}$ on $\mathrm{Cu}(110)$, and (d) is obtained from $2 \mathrm{ML} \mathrm{CH}_{3} \mathrm{Br}$ on $\mathrm{Cu}(110)$-I. In the spectra (a)-(c), the $\mathrm{CH}_{3}$ signal is collected along the surface normal, while in (d) the signal is collected $20^{\circ}$ from the normal- in all these cases, these angles are the direction of maximum $\mathrm{CH}_{3}$ yield.

When the thickness of the $n$-hexane "barrier" layer is varied for a fixed submonolayer of $\mathrm{CH}_{3} \mathrm{Br}$, the $\mathrm{CH}_{3}$ yield is found to vary as shown in Fig. 4. From temperature programmed desorption (TPD) measurements made for $n$-hexane on $\mathrm{Cu}(110)$ and $\mathrm{Cu}(100)$, the completion of the first monolayer occurs at approximately $4.5 \mathrm{~L}$ $n$-hexane dose. If it is assumed that the sticking coefficient is roughly constant for subsequent layers under our conditions (borne out by TPD measurements), we observe from Fig. 4 that very little $\mathrm{CH}_{3}$ yield is obtained for 2ML or less of $n$-hexane. The $\mathrm{CH}_{3}$ yield in Fig. 4 is observed to increase significantly as the $n$-hexane coverage increases beyond $2 \mathrm{ML}$ and reaches a maximum at approximately $4 \mathrm{ML} n$-hexane thickness. At coverages beyond $4 \mathrm{ML}$, the $\mathrm{CH}_{3}$ yield is found to decrease rapidly. Similar measurements made using the $\mathrm{Cu}(100)$ substrate showed essentially identical behavior- little or no $\mathrm{CH}_{3}$

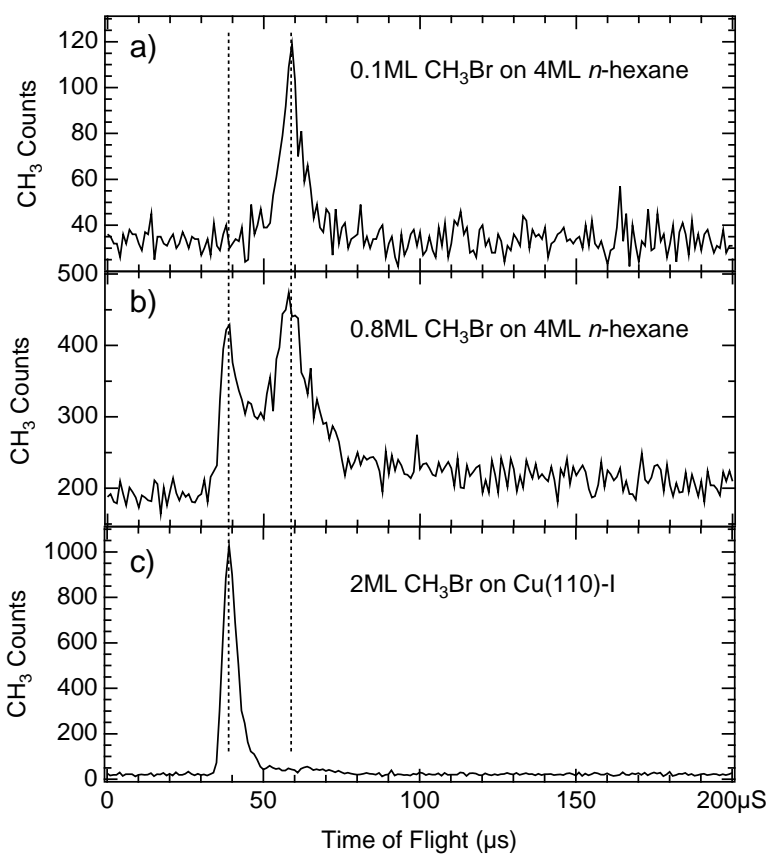

FIG. 3: Time-of-flight spectra for adsorbed $\mathrm{CH}_{3} \mathrm{Br}$ obtained using $193 \mathrm{~nm}$ light. In (a) the signal is from $0.1 \mathrm{ML} \mathrm{CH}_{3} \mathrm{Br}$ on top of $4 \mathrm{ML} n$-hexane on the $\mathrm{Cu}(110)$ substrate, and shows a single TOF feature due to image state mediated photodissociation. In spectrum (b), the $\mathrm{CH}_{3} \mathrm{Br}$ coverage is increased to $0.8 \mathrm{ML}$, and two TOF features are seen- the same image state mediated dissociation as for (a) at $59 \mu \mathrm{s}$ flight time and a feature due to direct photodissociation at $39 \mu \mathrm{s}$. As a comparison, (c) shows a TOF spectrum for $\mathrm{CH}_{3} \mathrm{Br}$ on $\mathrm{Cu}(110)-\mathrm{I}$ in which the direct photodissociation process dominates and there is no image state mediated dissociation visible.

yield from $2 \mathrm{ML}$ or less of $n$-hexane and a rapid rise in yield to a maximum near $4 \mathrm{ML} n$-hexane coverage. The decreasing $\mathrm{CH}_{3}$ yield for film thicknesses beyond $4 \mathrm{ML}$ is not due to changes in the $\mathrm{CH}_{3}$ angular distribution, as substantial angular changes were not observed. It is possible that the adsorbed submonolayer is increasingly subsumed in the $n$-hexane layers for higher coverage (e.g. at defects), though we do not see changes in the TOF spectrum peak shapes (i.e. translational energy distribution) or angular distributions. When $\mathrm{Cu}(100)-\mathrm{I}$ or $\mathrm{Cu}(100)-\mathrm{Cl}$ substrates are used, there are much lower yields (e.g. see Fig. 2(b)) but the coverage for maximum $\mathrm{CH}_{3}$ yield is observed to occur in the range of $1-2 \mathrm{ML} n$-hexane.

In studies of image state lifetimes of similar molecular systems using fs-2PPE, it is found that the lifetime increases rapidly (roughly exponentially) with increasing layer thickness. The $\mathrm{Cu}(110)$ substrate used in the present work has no surface bandgap at the image state energies, so the decay of the populated image states will be dominated by the electron tunneling through the $n$ hexane barrier. In contrast, the $\mathrm{Cu}(100)$ surface has a prominent s-p bandgap around $\bar{\Gamma}$ for energies relevant to the image states. That we observe essentially identi- 


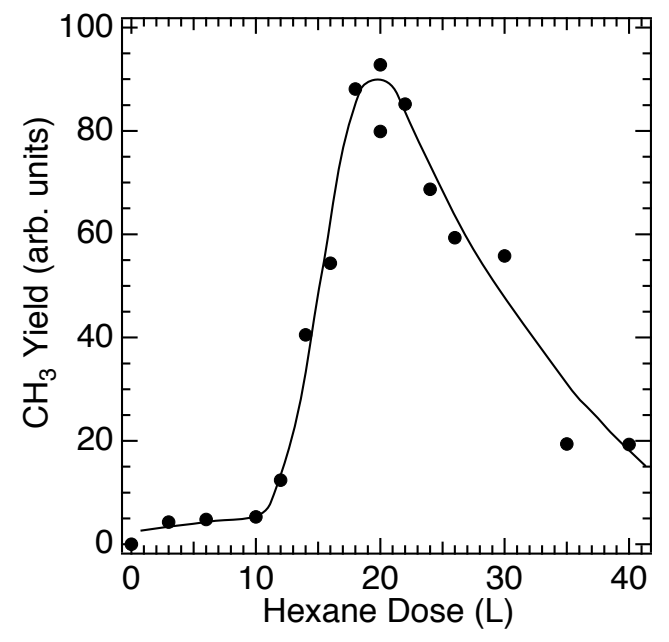

FIG. 4: Measured yield of $\mathrm{CH}_{3}$ from the 248nm photolysis of $0.1 \mathrm{ML} \mathrm{CH}_{3} \mathrm{Br}$ on top of a varying thickness of $n$-hexane on $\mathrm{Cu}(110)$. These data points are obtained from a series of individual TOF spectra, each the result of 1000 laser pulses, obtained in the surface normal direction. The solid curve is shown as a guide to the eye.

cal photodissociation behavior on both surfaces indicates that the metal surface intrinsic bandgap is not playing a significant role in these experiments. The low yield of $\mathrm{CH}_{3}$ for $2 \mathrm{ML}$ or less of $n$-hexane suggests that the image state lifetimes are too short for the image state electrons to localize on the $\mathrm{CH}_{3} \mathrm{Br}$ adsorption sites, and that for 3 to $4 \mathrm{ML} n$-hexane coverage, the probability to locate and dissociate the $\mathrm{CH}_{3} \mathrm{Br}$ becomes favorable. Another possibility is that the dissociating $\mathrm{CH}_{3} \mathrm{Br}^{-}$temporary anion is quenched more rapidly for these thin $n$-hexane films, presumably by overlap with unoccupied metal states (i.e. autodetachment). Experience from previous DEA photodissociation experiments would suggest that this is likely more important on the single monolayer thickness films. The optimal $\sim 4 \mathrm{ML} n$-hexane thickness for DEA from co-adsorbed $\mathrm{CH}_{3} \mathrm{Br}$ seems to represent a compromise between the formation and population of the image state and the image state lifetime. For thicker $n$ hexane films $(>4 \mathrm{ML})$ the decreased $\mathrm{CH}_{3}$ yield is most likely due to a decrease in the initial population of the $n$-hexane-vacuum interface image state, which becomes increasingly less probable as the requisite photoelectrons generated in the metal substrate cannot tunnel through the thick $n$-hexane layers.

One more possibility to consider is that the $\mathrm{CH}_{3} \mathrm{Br}$ dissociation is due to DEA from an interface electron state inside the $n$-hexane layer (similar to states identified in Ar thin films 24]), and the rapid rise in the 2ML$4 \mathrm{ML}$ region is due to the exclusion of this state from the thinner films. Sustaining such a state requires a surface bandgap for the metal substrate at the relevant energies and we observe no differences between the behavior on $\mathrm{Cu}(100)$ (which has such a gap at $\bar{\Gamma}$ ) and $\mathrm{Cu}(110)$ (which does not). Also when experiments were performed on $\mathrm{Cu}(100)-\mathrm{I}$ or $\mathrm{Cu}(100)-\mathrm{Cl}$ the maximum signal was seen for much thinner films (1-2ML) so we do not believe that such an interface electron state in $n$-hexane is responsible for the observed dissociation.

If the observed photodissociation of adsorbed $\mathrm{CH}_{3} \mathrm{Br}$ is mediated by an image state electron intermediate, then one expectation would be that this photodissociation would be very efficient for even a small submonolayer of the dissociating species. The image state electron at the thin film-vacuum interface, though constrained in the surface normal direction, would be expected to be mobile parallel to the surface as described above. The data of Fig. 5 support this notion- there is a very steep increase in the observed $\mathrm{CH}_{3}$ yield for submonolayer $\mathrm{CH}_{3} \mathrm{Br}$ on the 4ML $n$-hexane on $\mathrm{Cu}(110)$ system using $248 \mathrm{~nm}$ light. From TPD and photochemistry experiments on clean $\mathrm{Cu}(110)$, we have found that a $6.5 \mathrm{~L}$ dose of $\mathrm{CH}_{3} \mathrm{Br}$ corresponds to one monolayer coverage, and for adsorption on $n$-hexane, one would anticipate that the sticking coefficient would be, at best, the same, so that the observed increase in the region of $0-2 \mathrm{~L}$ dose corresponds to roughly $0-0.3 \mathrm{ML}$ coverage range. At higher coverages (above $\sim 0.5 \mathrm{ML}$ ) the $\mathrm{CH}_{3}$ angular distribution becomes broader, so the observed roughly constant yield at higher doses measured in the surface normal direction in Fig. 4 is not entirely reflective of the total yield, however the steep increase in yield at low coverage is not affected by this. From image state studies at clean metal surfaces with intrinsic surface bandgaps (e.g. using inverse photoemission), a sensitivity of the image state to surface contamination has been noted [3]. This surface contamination sensitivity does not seem to carry over to the image states created at thin $n$-hexane surfaces, the characteristic image state dissociation is observable even at monolayer coverages of the dissociating species (for example, see Fig. 3(b)).

In addition to $\mathrm{CH}_{3} \mathrm{Br}$, we have also observed the same image state mediated dissociation occur for adsorbed $\mathrm{CH}_{3} \mathrm{I}$ submonolayers. $\mathrm{CH}_{3} \mathrm{I}$ has a larger cross section for DEA in the gas-phase than $\mathrm{CH}_{3} \mathrm{Br}$ does, with a peak cross section of $>100 \times 10^{-16} \mathrm{~cm}^{2}$ for $E<60 \mathrm{meV}[11$. In the adsorbed state, $\mathrm{CH}_{3} \mathrm{I}$ has a large DEA cross section for very low energy electrons $\left(\sigma=6.8 \times 10^{-16} \mathrm{~cm}^{2}\right.$ at $\mathrm{E} \sim 0 \mathrm{eV}$ when adsorbed on 10ML of $\mathrm{Kr}$ ) [17, with the peak DEA cross section having apparently shifted to an energy below the vacuum level due to image interactions with the surface. A TOF spectrum obtained at $248 \mathrm{~nm}$ for $0.1 \mathrm{ML} \mathrm{CH}_{3} \mathrm{I}$ on $4 \mathrm{ML} n$-hexane on $\mathrm{Cu}(110)$ is shown in Fig. 6(a). In this case, the $\mathrm{CH}_{3} \mathrm{I}$ TOF distribution is narrower than that seen for CT-DEA of adsorbed $\mathrm{CH}_{3} \mathrm{I}$ in other cases. If the $\mathrm{CH}_{3} \mathrm{I}$ coverage is increased, and ppolarized $248 \mathrm{~nm}$ light is used, photodissociation by both CT-DEA and by direct photodissociation can be seen (Fig. 6(b)). Similar to the case for $\mathrm{CH}_{3} \mathrm{Br}$ at $193 \mathrm{~nm}$, direct photodissociation can be seen in the small fast peak at $45 \mu \mathrm{s}$ flight time. Another contrast can be seen in Fig. 6(c) which shows a TOF spectrum obtained from 


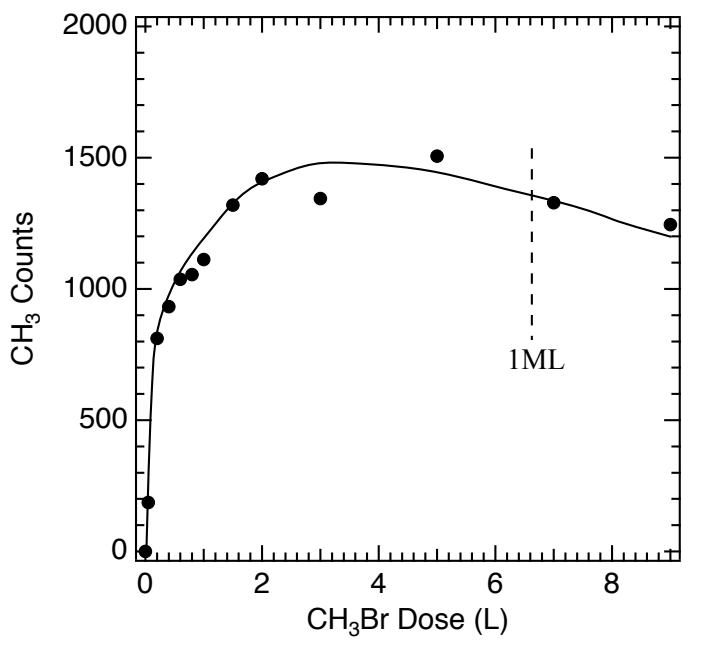

FIG. 5: Yield of $\mathrm{CH}_{3}$ from photolysis of varying amounts of $\mathrm{CH}_{3} \mathrm{Br}$ adsorbed on $\sim 4 \mathrm{ML} n$-hexane on $\mathrm{Cu}(110)$. The measured counts are detected in the surface normal direction from 1000 pulses of $248 \mathrm{~nm}$ light. From TPD experiments on clean $\mathrm{Cu}(110)$, one nominal monolayer of $\mathrm{CH}_{3} \mathrm{Br}$ corresponds to $\sim 6.5 \mathrm{~L} \mathrm{CH}_{3} \mathrm{Br}$ dose. The large increase in $\mathrm{CH}_{3}$ yield corresponds to the range $0-0.3 \mathrm{ML} \mathrm{CH}_{3} \mathrm{Br}$ coverage.

$2 \mathrm{ML} \mathrm{CH}_{3} \mathrm{I}$ that is well ordered on a $\mathrm{Cu}(110)$-I substrate at $308 \mathrm{~nm}(h \nu=4.02 \mathrm{eV})$ using s-polarized light. Under these conditions, the TOF spectrum is dominated by the DEA mechanism [19] from subvacuum level photoelectrons. The TOF spectrum for the $\mathrm{CH}_{3} \mathrm{I}$ on $n$-hexane is noticeably narrower than that obtained at the longer wavelength on $\mathrm{Cu}(110)$-I.

Although for $\mathrm{CH}_{3} \mathrm{I}$ we observe that the $\mathrm{CH}_{3}$ TOF distributions are not as narrow as those found for $\mathrm{CH}_{3} \mathrm{Br}$, they are still narrower than those observed in other situations. One possible explanation for the slightly broader $\mathrm{CH}_{3}$ TOF distribution for $\mathrm{CH}_{3} \mathrm{I}$, as compared to $\mathrm{CH}_{3} \mathrm{Br}$ is that $\mathrm{CH}_{3} \mathrm{I}$ does have a substantially larger intrinsic (i.e. not image state mediated) DEA cross section, so that there may be an additional contribution to the observed TOF signal from a broader range of DEA photoelectron energies. That DEA can dominate the $\mathrm{CH}_{3} \mathrm{I}$ dissociation at $248 \mathrm{~nm}$ is in itself surprising, since the cross section for direct photolysis at this wavelength is quite large. Similar to the case for $\mathrm{CH}_{3} \mathrm{Br}$ at $193 \mathrm{~nm}$, for small submonolayer coverages of $\mathrm{CH}_{3} \mathrm{I}$ at $248 \mathrm{~nm}$, the image mediated DEA dominates the photodissociation. Direct photolysis is found to be increasingly important at higher $\mathrm{CH}_{3} \mathrm{I}$ coverage, especially when p-polarized light is present since the oriented $\mathrm{CH}_{3} \mathrm{I}$ has a large cross section in this case 19. It has also been found that all of the same general findings made for $\mathrm{CH}_{3} \mathrm{Br}$ are also found for $\mathrm{CH}_{3} \mathrm{I}$ - the pronounced yield variation for varying $n$ hexane spacer thickness, the large observed yields at low $\mathrm{CH}_{3} \mathrm{I}$ coverage and very similar angular distributions of the $\mathrm{CH}_{3}$ photofragments. The findings made for $\mathrm{CH}_{3} \mathrm{I}$ complement those made for $\mathrm{CH}_{3} \mathrm{Br}$ and lend further sup-

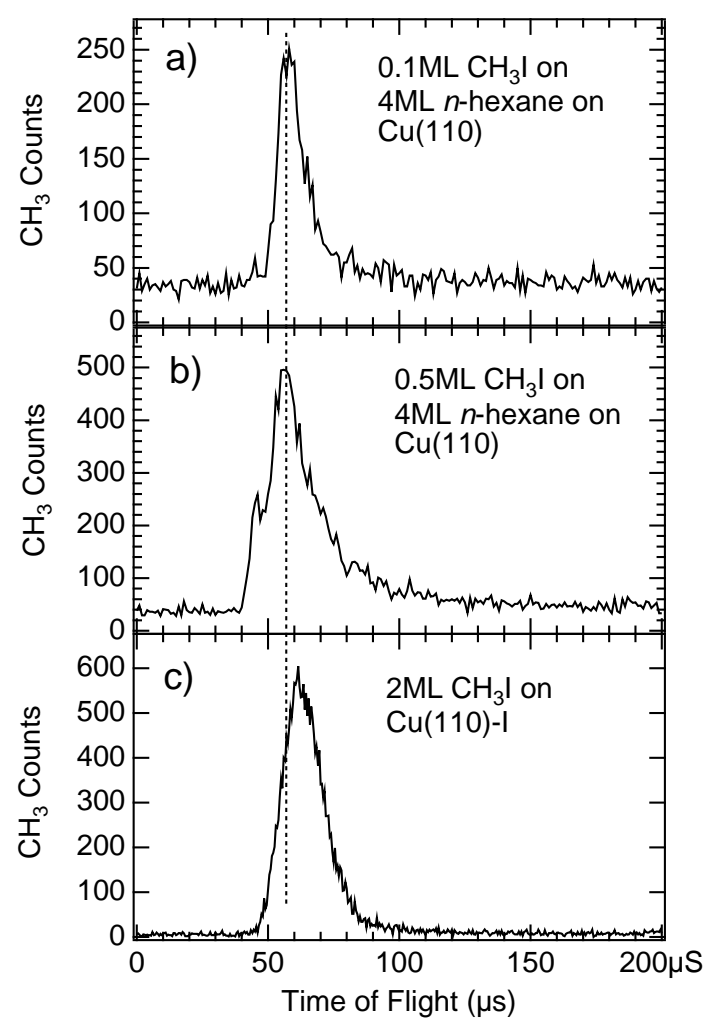

FIG. 6: Time-of-flight spectra from $\mathrm{CH}_{3} \mathrm{I}$ : (a) from a submonolayer on $4 \mathrm{ML} n$-hexane (248nm s-polarized light), (b) from $0.5 \mathrm{ML} \mathrm{CH}_{3} \mathrm{I}$ on $4 \mathrm{ML} n$-hexane (248nm p-polarized light) and (c) from $2 \mathrm{ML} \mathrm{CH}_{3} \mathrm{I}$ on $\mathrm{Cu}(110)$-I obtained using spolarized $308 \mathrm{~nm}$ light.

port to the proposed image state mediated dissociation mechanism.

The energetics of the dissociation by low energy electrons and the contrast between the case for $\mathrm{CH}_{3} \mathrm{Br}$ and $\mathrm{CH}_{3} \mathrm{I}$ are further illustrated by the TOF data shown in Fig. 7. For rapid dissociation via DEA for a free $\mathrm{CH}_{3} \mathrm{X}$ molecule, the translational energy of the $\mathrm{CH}_{3}$ fragment can be understood from:

$$
\begin{aligned}
T_{C H_{3}}=\frac{m_{X}}{m_{C H_{3} X}}\{ & \left\{E_{e^{-}}+E A(X)-D_{0}(C-X)\right. \\
& \left.+\Delta E_{\text {solvation }}\left(X^{-}\right)-E_{\text {int }}\left(C H_{3}\right)\right\}
\end{aligned}
$$

where the mass ratio accounts for momentum conservation for a dissociating free molecule, $E_{e^{-}}$is the kinetic energy of the incident electron, $E A(X)$ is the electron affinity of the halogen atom $(E A(B r)=3.36 \mathrm{eV}, E A(I)=$ $3.06 \mathrm{eV}) 25, D_{0}(C-X)$ is the methyl-halogen bond dissociation energy from the ground state $\left(D_{0}(C-B r)=\right.$ $\left.3.05 \mathrm{eV}, D_{0}(C-I)=2.48 \mathrm{eV}\right) 25, \Delta E_{\text {solvation }}\left(X^{-}\right)$is the additional stabilization energy for the halogen anion at the surface and $E_{\text {int }}\left(\mathrm{CH}_{3}\right)$ accounts for internal excitation of the $\mathrm{CH}_{3}$ fragment upon dissociation. If we assume that $E_{\text {int }}\left(\mathrm{CH}_{3}\right)$ is similar for both $\mathrm{CH}_{3} \mathrm{Br}$ and $\mathrm{CH}_{3} \mathrm{I}$ and is near zero for the fastest $\mathrm{CH}_{3}$ fragments, 


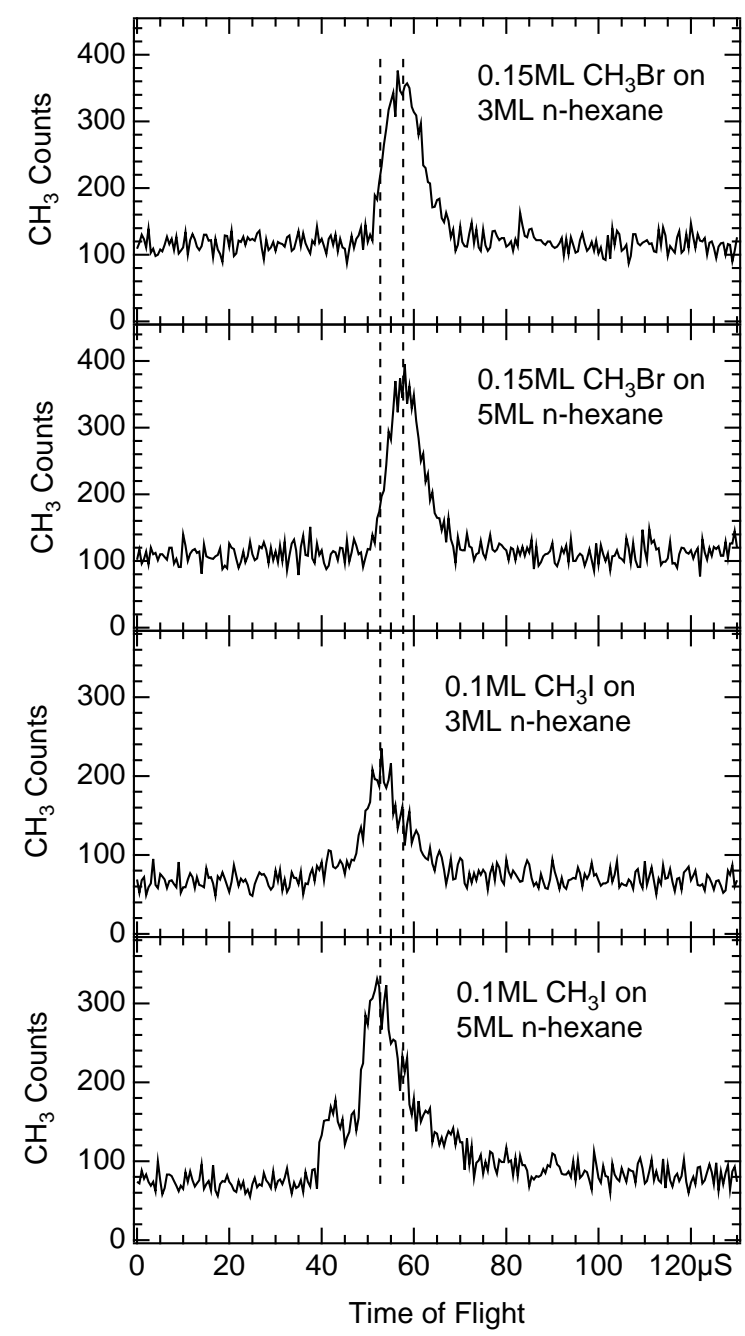

FIG. 7: Higher resolution time-of-flight spectra (0.5 $\mu \mathrm{s}$ bins) for submonolayers of $\mathrm{CH}_{3} \mathrm{Br}$ and $\mathrm{CH}_{3} \mathrm{I}$ on $3 \mathrm{ML}$ and $5 \mathrm{ML} n$ hexane thin films obtained by summing data three independent scans. These spectra were obtained using unpolarized $248 \mathrm{~nm}$ light and a $\mathrm{Cu}(100)$ substrate. There is no detectable shift in the TOF spectrum for the DEA features for differing $n$-hexane film thicknesses, but there is a shift of $5.0 \mu$ s between the DEA features for $\mathrm{CH}_{3} \mathrm{Br}$ and $\mathrm{CH}_{3} \mathrm{I}$.

then $E_{e^{-}}+\Delta E_{\text {solvation }} \approx 1.0 \mathrm{eV}$ gives $T_{C H_{3}}$ consistent with the observed values. This seems reasonable given that one would expect $\Delta E_{\text {solvation }}$ to be of the order of $0.8 \mathrm{eV}[26]$, by analogy with electron scattering measurements for $\mathrm{N}_{2}$ on thin rare gas films and $E_{e^{-}} \approx 0.2 \mathrm{eV}$ is reasonable. Based on these values, the expected TOF differences for $\mathrm{CH}_{3}$ from $\mathrm{CH}_{3} \mathrm{Br}$ and $\mathrm{CH}_{3} \mathrm{I}$ would be roughly $6.0 \mu \mathrm{s}$, which is slightly larger than the $5.0 \mu \mathrm{s}$ shift we observe in Fig. 7. It would be expected that the solvation energies for the $\mathrm{Br}^{-}$will be somewhat larger than that for $\mathrm{I}^{-}$, though the precise magnitude of this difference is not known for the halogen anions at a hexane surface- a difference of $\sim 0.04 \mathrm{eV}$ would make the values consistent with the observed flight time differences.
Another interesting observation from Fig. 7 is that there is no detectable shift between the TOF spectra from $3 \mathrm{ML}$ and $5 \mathrm{ML} n$-hexane coverages for either $\mathrm{CH}_{3} \mathrm{Br}$ of $\mathrm{CH}_{3} \mathrm{I}$. This implies that $E_{e^{-}}+\Delta E_{\text {solvation }}$ is essentially constant over the range of $n$-hexane coverages used for $\mathrm{CH}_{3} \mathrm{X}$ dissociation. For example, between $3 \mathrm{ML}$ and $5 \mathrm{ML} n$-hexane films the $n=1$ image state energy rises (from calculations such as that shown in Fig. 11) from $-0.40 \mathrm{eV}$ to $-0.33 \mathrm{eV}$ but $\Delta E_{\text {solvation }}$ would be expected to decrease 26 by $\sim 0.090 \mathrm{eV}$ due to the increased anion to metal surface distance, for an expected change in TOF of $+0.5 \mu \mathrm{s}$. Within the resolution of our TOF spectra we do not detect any shifts in either the leading edge or the centroid of the TOF distributions. It is also worth noting that while the contribution of higher energy $n=2$ image state electrons would yield significantly faster $\mathrm{CH}_{3}$ fragments (by $\sim 6.8 \mu \mathrm{s}$ ), the relative number of these is much smaller [10] than for the $n=1$ image state, which seems to be primarily due to the much smaller spatial overlap of the $n=2$ wavefunction with the population of hot photoelectrons that are transported to the metal interface.

\section{CONCLUSIONS}

The photodissociation of $\mathrm{CH}_{3} \mathrm{Br}$ and $\mathrm{CH}_{3} \mathrm{I}$ adsorbed on top of thin $n$-hexane layers has been found to be caused by hot photoelectrons generated in the metal substrate. The nascent photoelectron energy distribution is modified by the $n$-hexane layers and some of these photoelectrons are temporarily trapped at the $n$-hexane-vacuum interface in the $n=1$ image state. These image state electrons can interact with the co-adsorbed $\mathrm{CH}_{3} \mathrm{X}$ and cause dissociation via the DEA mechanism. The $\mathrm{CH}_{3}$ photofragments detected in the TOF experiment have a translational energy distribution that is insensitive to the incident photon energy and the yields of $\mathrm{CH}_{3}$ are indicative of the image state mediated mechanism. This mechanism is likely to be viable for a number of molecular systems, as a wide variety of molecules have the requisite negative electron affinity and bandgap to create such image states, and there are also many molecules which undergo various chemical processes due to interactions with very low energy electrons.

\section{Acknowledgements}

The author would like to thank the Natural Sciences and Engineering Research Council (NSERC) of Canada for financial support of this work. Thanks also to Jeff Hnybiba, Robert Vogt and Masresha Berhanu, who provided assistance with the experiments as a part of their NSERC Undergraduate Student Research Award tenure in the laboratory. 
[1] F. Weik, A. de Meijere, and E. Hasselbrink, J. Chem. Phys. 99, 682 (1993).

[2] P. J. Rous, Phys. Rev. Lett. 74, 1835 (1995).

[3] M. Donath and K. Ertl, Surf. Sci. Lett. 262, L49 (1992).

[4] M. W. Cole and M. H. Cohen, Phys. Rev. Lett. 23, 1238 (1969).

[5] C. C. Grimes and T. R. Brown, Phys. Rev. Lett. 32, 280 (1974).

[6] W. Berthold, F. Rebentrost, P. Feulner, and U. Höfer, Appl. Phys. A. 78, 131 (2004).

[7] C. B. Harris, N.-H. Ge, R. L. Lingle Jr., J. D. McNeill, and C. M. Wong, Annu. Rev. Phys. Chem. 48, 711 (1997).

[8] A. Hotzel, K. Ishioka, E. Knoesel, M. Wolf, and G. Ertl, Chem. Phys. Lett. 285, 271 (1998).

[9] A. Hotzel, G. Moos, K. Ishioka, M. Wolf, and G. Ertl, Appl. Phys. B 68, 615 (1999).

[10] R. L. Lingle Jr, N.-H. Ge, R. E. Jordan, J. D. McNeill, and C. B. Harris, Chem. Phys. 205, 191 (1996).

[11] A. Schramm, I. I. Fabrikant, J. M. Weber, E. Leber, M.W. Ruf, and H. Hotop, J. Phys. B: At. Mol. Opt. Phys. 32, 2153 (1999).

[12] R. S. Wilde, G. A. Gallup, and I. I. Fabrikant, J. Phys. B: At. Mol. Opt. Phys. 33, 5479 (2000).

[13] X.-L. Zhou, X.-Y. Zhu, and J. M. White, Surf. Sci. Rep. 13, 73 (1991).
[14] V. A. Ukraintsev, T. J. Long, and I. Harrison, J. Chem. Phys. 96, 3957 (1992).

[15] S. J. Dixon-Warren, D. V. Heyd, E. T. Jensen, and J. C. Polanyi, J. Chem. Phys. 98, 5954 (1993).

[16] L. Sanche, A. D. Bass, P. Ayotte, and I. I. Fabrikant, Phys. Rev. Lett. 75, 3568 (1995).

[17] E. T. Jensen and L. Sanche (2007), submitted.

[18] P. Ayotte, J. Gamache, A. D. Bass, I. I. Fabrikant, and L. Sanche, J. Chem. Phys. 106, 749 (1997).

[19] E. T. Jensen, J. Chem. Phys. 123, 204709 (2005).

[20] C. C. Johnson and E. T. Jensen, J. Chem. Phys. 112, 5919 (2000).

[21] B. V. Andryushechkin, K. N. Eltsov, and V. M. Shevlyuga, Surf. Sci. 584, 278 (2005).

[22] E. T. Jensen, J. Phys.: Condens. Matter 18, S1345 (2006).

[23] G. N. A. Van Veen, T. Baller, and A. E. De Vries, Chem. Phys. 92, 59 (1985).

[24] M. Rohleder, W. Berthold, J. Güdde, and U. Höfer, Phys. Rev. Lett. 94, 017401 (2005).

[25] D. R. Lide, ed., CRC Handbook of Chemistry and Physics (Taylor and Francis, 2006), 87th ed.

[26] M. Michaud and L. Sanche, J. Elec. Spectrosc. Relat. Phenom. 51, 237 (1990). 